\title{
MODELO DE SATISFAÇÃO ACSI MODIFICADO NO SETOR DE TELEFONIA MÓVEL
}

RESUMO

O propósito do artigo consiste na validação do modelo de satisfação American Customer Satisfaction Index - ACSI modificado - no âmbito do setor de telefonia móvel. 0 modelo original foi modificado com a inclusão do construto conveniência e da fase qualitativa na pesquisa. 0 método abrangeu duas fases: exploratória e descritiva. A primeira teve por finalidade a adaptação do modelo ao setor pesquisado, sendo utilizada a técnica dos grupos de foco. Na fase quantitativa foi consultada uma amostra de 606 usuários de celular: clientes das três operadoras atuantes no estado de Minas Gerais. A técnica de modelagem de equações estruturais foi utilizada para análise de plausibilidade do modelo proposto. Os resultados sugerem os seguintes relacionamentos do modelo: Qualidade $\rightarrow$ Valor Percebido, Qualidade $\rightarrow$ Satisfação, Valor Percebido $\rightarrow$ Satisfação, e Satisfação $\rightarrow$ Leal dade.

\section{Andréia Cássia de Moura}

Faculdade Batista de Minas Gerais

e Faculdade Estácio de Sá

\section{Carlos Alberto Gonçalves}

UFMG

\begin{abstract}
This work aims to validate a model of satisfaction, the modified American Customer Satisfaction Index (ACSI) on the mobile telecommunication area in Brazil. In this research, the original ACSI model was modified by the inclusion of a construct, the Convenience and also due to the qualitative phase. The exploratory phase was conducted in order to adapt the model to the researched sector and the technique used was focus group. In the quantitative phase a sample of 606 users of cell phone was interviewed: clients of the three mobile telecommunication companies of M inas Gerais, in Brazil. D ata statistical treatment was supported by Structural Equations M odeling to analyze plausibility of the new proposed model. The results indicated towards a proposed model validity. The positive relationship of the following variables was supported by data analysis: Quality $\rightarrow$ Perceived Value, Quality $\rightarrow$ Satisfaction, Value $\rightarrow$ Satisfaction, and Satisfaction $\rightarrow$ Loyalty.
\end{abstract}

PALAVRAS-CHAVE Modelo de satisfação ACSI modificado, relacionamento entre variáveis, modelagem de equações estruturais, validação de modelo, telefonia móvel.

KEYMORDS M odified satisfaction model ACSI, relationship among variables, structural equations modeling, model validation, mobile telecommunication. 


\section{INTRODUÇÃO}

Com a abertura do mercado, a competição no setor brasileiro de telefonia se tornou acirrada e tem impulsionado novas configurações e a qualificação da oferta de serviços. Além disso, as empresas de telecomunicações se defrontam com um ambiente incerto e um alto grau de obsolescência da tecnologia, o que exige respostas rápidas e adequadas para sua sobrevivência.

Após a privatização do sistema Telebrás, em 1998, ocorreram transformações marcadas pelo crescente aumento do número de linhas fixas instaladas, pela crescente evolução do número de aparel hos celulares e pela queda no preço dos tel efones devido ao aumento da oferta (Oliveira, 2000). A liberação de concessões pela Agência Nacional de Telecomunicações (Anatel) em 2002 fez surgir no mercado um vasto número de fornecedores nacionais e estrangei ros que concorrem entre si.

N esse novo cenário desponta uma questão fundamental que diz respeito à avaliação dos clientes quanto à oferta de serviços dos prestadores, isto é, o quanto 0 serviço tem atendido bem às necessidades dos clientes. $N$ esse sentido, um indicador de satisfação global seria muito útil por refletir as opiniões dos clientes acerca dos serviços, além de permitir outras vantagens.

0 modelo American Customer Satisfaction Index (ACSI) proposto por Fornell et al. (1996) pretende oferecer um índice de mensuração comparável para o conceito ( construto) "satisfação do cliente", além de indicar as relações de tal construto com seus principais antecedentes (expectativas, qualidade percebida, valor percebido) e conseqüentes (leal dade e reclamações). Assim, 0 objetivo do artigo é verificar empiricamente o ajustamento de um modelo de satisfação baseado no modelo ACSI ao setor de telefonia móvel. Esse modelo foi denominado ACSI modificado, pois se adicionou a ele um construto antecedente da satisfação do consumidor - a conveniência -, além de modificações decorrentes da fase qual itativa da pesquisa. Com isso, pretendeu-se contribuir no sentido de compreender a satisfação do cliente em um setor de destaque para a economia nacional, al ém de entender as relações da satisfação com seus principais antecedentes e conseqüentes, sendo relevante ampliar as poucas iniciativas de estudo do tema no Brasil.

\section{REFERENCIAL TEÓRICO}

\section{Satisfação do consumidor}

A satisfação do consumidor pode ser contextualizada como integrante da teoria do comportamento do consumidor. Iniciado com o trabal ho de Cardozo (1965) , 0 campo de pesquisa sobre a satisfação do consumidor evoluiu com crescente número de estudos, tendo em vista sua grande relevância para o marketing. Segundo Churchill e Surprenant (1982), a importância da satisfação está na geração de lucros por meio da realização das necessidades e desejos dos consumidores.

A satisfação foi definida por Oliver (1997) como a resposta da realização do consumidor, no sentido de avaliar se as características do bem ou serviço atendem às suas necessidades, proporcionando um nível prazeroso de consumo. Essa avaliação ocorre quando o consumidor compara os benefícios recebidos do bem ou serviço com suas expectativas.

De acordo com Boulding et al. (1993), a conceituação de satisfação encontra duas dimensões: transação específica e acumulada. A primeira envolve 0 julgamento individual da experiência com um produto ou serviço, sendo uma visão particular e de curto prazo. Na segunda, a satisfação é caracterizada como um construto abstrato que descreve a experiência total de consumo com um produto ou serviço. Então, para o monitoramento do desempenho atual e de longo prazo da empresa de acordo com a avaliação dos clientes, essa abordagem é mais aplicável. Sendo assim, neste estudo a satisfação foi tratada sob a perspectiva cumulativa em consonância com os autores do modelo ACSI.

\section{0 modelo ACSI}

N uma perspectiva histórica, o primeiro índice de satisfação do cliente de âmbito real mente nacional foi 0 Customer Satisfaction Barometer (CSB), desenvolvido em 1989 (F ornell, 1992). O Índice de satisfação do cliente norte-americano foi introduzido em 1994, contendo informações sobre 40 ramos de atividades, de sete grandes grupos da economia dos Estados Unidos (Fornell et al., 1995). Posteriormente, a N ova Zelândia começou a trabalhar com índices de satisfação do cliente, ao passo que a União Européia recomendou a criação de tal tipo de índice em seus países membros (Fornell et al., 1996). Testes pilotos têm sido também implementados em Taiwan, Coréia e Brasil (Anderson e Fornell, 2000).

O modelo ASCI proposto por Fornell et al. (1996) trata a "satisfação global do cliente" como construto central do modelo, cujos relacionamentos com seus antecedentes (expectativas, qualidade e valor percebidos pelo cliente) e conseqüentes (reclamações e leal- 
dade do cliente) são hipotetizados no modelo. A satisfação global do cliente não pode ser medida diretamente, sendo uma variável latente que requer múltiplos indicadores na sua mensuração (veja a Figura 1). Como resultado da operacionalização desse modelo é encontrado um índice da variável latente da satisfação global do cliente, que permitirá comparações entre organizações, ramos de atividades, setores e nações.

Com respeito aos antecedentes de satisfação, são preconizados os seguintes relacionamentos: a) as expectativas compõem um determinante da satisfação. A suposição do modelo é de que as expectativas do cliente sejam positivamente relacionadas à qualidade percebida e, em decorrência, ao valor percebido; b) supõe-se que a qualidade percebida tenha um efeito direto e positivo sobre a satisfação global dos clientes e um efeito positivo sobre o valor percebido; e c) para o construto de valor percebido é predita uma associação positiva entre este e a satisfação do cliente.

Quanto aos conseqüentes da satisfação, o modelo supõe que um aumento da satisfação global do cliente deva reduzir a incidência de reclamações e aumentar a leal dade do cliente. Para os autores do modelo ACSI, a leal dade está ligada à probabilidade de recompra pel o cliente, além de considerar a tol erância ao preço como um fator componente. 0 relacionamento final do modelo é entre reclamações do cliente e sua leal dade. 0 sinal desse relacionamento depende dos sistemas de serviço ao cliente e de solução de reclamações dada pelo prestador (Fornell, 1992). Quando as respostas do prestador são favoráveis ao cliente, o relacionamento é positivo. De outro modo, o relacionamento será negativo.

No Brasil, os primeiros testes empíricos do modeIo ACSI podem ser encontrados nos trabalhos de Urdan e Rodrigues (1998), M archetti e Prado (2001) e Gonçalves Filho, Guerra e Moura (2003). 0 primeiro trabal ho consistiu na avaliação do ajustamento do modelo no caso de clientes da indústria automobilística.

Marchetti e Prado (2001) estudaram o setor el étrico brasileiro. Os autores, com base no modelo do índice de satisfação do consumidor norte-americano, propuseram um modelo de avaliação da satisfação do consumidor para esse setor. M ais especificamente, foi apresentada uma adaptação do modelo, sendo os antecedentes da satisfação - desconformidade percebida e valor percebido - e o conseqüente à fidelidade. A escala para os construtos adaptados - desconformidade percebida (fusão de expectativas e qualidade), valor percebido e fidelidade - foi elaborada e validada por meio dos atributos relevantes levantados nos grupos de discussão realizados na fase qualitativa.

Como resultado da aplicação desse modelo, o nível de satisfação dos consumidores tem sido mensurado

Figura 1 - Modelo ACSI original.

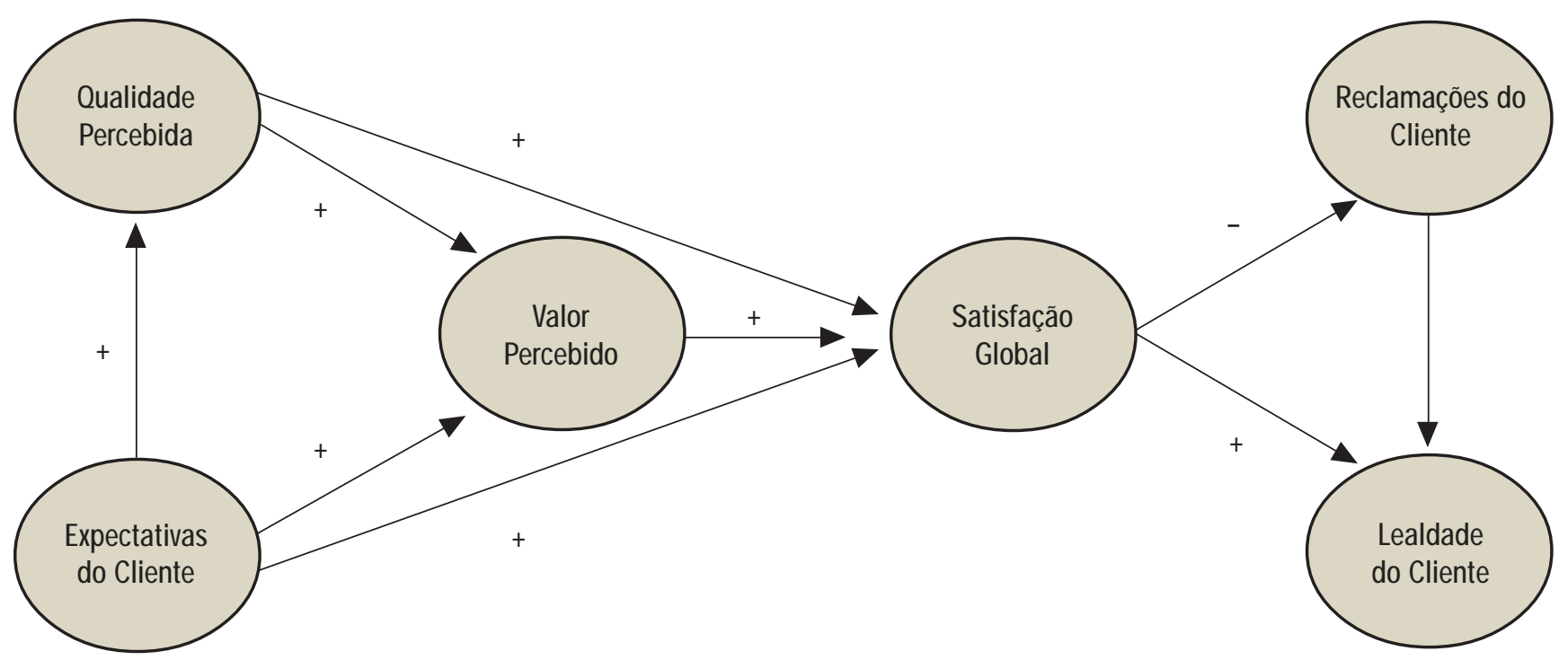

Fonte: Fornell et al. (1996, p. 8). 
por meio do Índice Aneel de Satisfação do Consumidor (IASC) desde 2000. 0 órgão regulamentador do setor, a Agência N acional de Energia Elétrica (Aneel), tem utilizado esse indicador para comparar o desempenho das concessionárias de energia elétrica brasileiras a partir da avaliação dos clientes.

Outra aplicação no Brasil encontra-se no trabal ho de Gonçalves Filho, Guerra e Moura (2003). Esses autores aplicaram o modelo ACSI em instituições de ensino superior, verificando um grande impacto da satisfação sobre a lealdade dos alunos. Observou-se a validade do modelo de uma forma geral, havendo rejeição das hipóteses concernentes aos vínculos entre as expectativas e os construtos val or e satisfação. É relevante dizer que os estudos de Marchetti e Prado (2001) sobre o ACSI consistiram numa referência importante para o presente artigo, já que a pretensão foi adaptar o modelo ACSI ao contexto do setor de telefonia móvel, levantando-se os indicadores dos construtos por meio de grupos de foco (fase qualitativa) e posterior aplicação dos questionários (fase quantitativa)

\section{MODELO HIPOTÉTICO DA PESQUISA}

0 modelo hipotético da pesquisa foi proposto com base no modelo ACSI e nos resultados da fase quali- tativa explicitados na seção seguinte, onde são também discutidas as modificações sugeridas. A suposição levantada para o model o proposto é de que a conveniência esteja ligada ao valor percebido, ou seja, que haja uma rel ação linear positiva entre esses construtos (veja a Figura 2). As relações do novo construto com os demais da cadeia nomológica foram identificadas por meio de grupo de discussão de especialistas da área de marketing. Tais relações também encontram respaldo direta ou indiretamente em artigos recentes (Berry, Seiders e Grewal, 2002; Brown, 1990).

Verificaram-se as seguintes hipóteses nulas:

- $\mathrm{H}_{01}$ : não há um relacionamento linear positivo significativo entre os construtos conveniência e val or percebido;

- $\mathrm{H}_{02}$ : não há um relacionamento linear positivo significativo entre os construtos qualidade percebida e valor percebido;

- $\mathrm{H}_{03}$ : não há um relacionamento linear positivo significativo entre os construtos qualidade percebida e satisfação global do cliente;

- $\mathrm{H}_{04}$ : não há um relacionamento linear positivo significativo entre os construtos val or percebido e satisfação global do cliente;

- $\mathrm{H}_{05}$ : não há um relacionamento linear positivo significativo entre os construtos satisfação global do cliente e sua lealdade ao fornecedor de serviços.

Figura 2 - Modelo ACSI modificado proposto final.

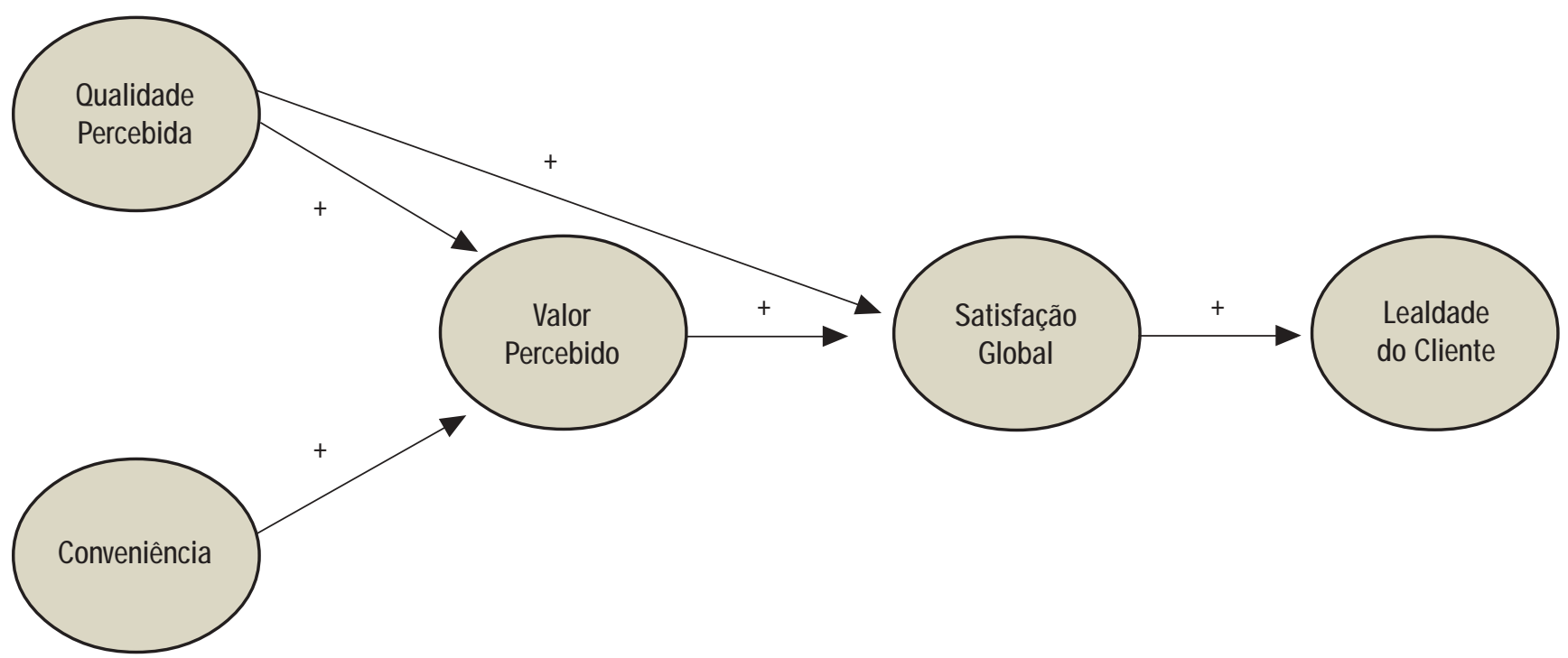

Fonte: Elaborado com base em Fornell et al. (1996) e grupos de foco. 


\section{PROCEDIMENTOS METODOLÓGICOS}

A presente pesquisa se inseriu na classificação de pesquisa conclusiva descritiva, uma vez que teve como objetivo testar hipóteses específicas e examinar relacionamentos (associações) entre construtos ( $M$ al hotra, 2001). As unidades de análise foram as empresas de telefonia móvel atuantes em M inas Gerais, num total de três operadoras. Para avaliar o modelo de satisfação do consumidor proposto pela pesquisa - ACSI modificado - foram consultados os usuários finais dos serviços de tel efonia móvel no âmbito de M inas Gerais, os quais constituíram as unidades de observação da pesquisa.

Para a realização da pesquisa utlizou-se o método survey, pois apresenta a vantagem de quantificação dos dados e a generalização dos dados para a população (Malhotra, 2001). Além disso, por ter sido o survey 0 método empregado pelos proponentes do modelo ACSI, ele foi utilizado para fins de comparação entre os modelos.

A metodologia baseada no modelo de Churchill (1979) mostrou-se adequada à solução do problema de pesquisa concernente à validação do modelo teórico proposto, visto que a pesquisa partiu de uma fase exploratória importante com o objetivo de gerar indicadores para os construtos em questão. Mais especificamente, 0 alcance dos indicadores permitiria a confirmação ou não dos indicadores originais, fazendo-se as adaptações necessárias a sua aplicação em outra realidade e em um setor específico.

A pesquisa foi então implementada com base nesse modelo, o qual implica a consecução de oito etapas, a saber: 1) especificar o domínio do construto; 2) gerar amostra de itens; 3 ) coletar dados na fase exploratória; 4) purificar/refinar medida; 5) coletar dados com 0 instrumento gerado na fase exploratória; 6) avaliar a confiabilidade; 7) avaliar a validade; e, 8) desenvolver normas, ou seja, utilizar medidas de comparação que sumarizam a distribuição de valores.

\section{Fase qualitativa/ exploratória}

Inicialmente foi realizada a fase qualitativa, objetivando o levantamento dos indicadores dos construtos propostos do modelo e a posterior el aboração do instrumento de pesquisa. 0 intuito final foi efetuar comparações entre os indicadores originais e fazer as adaptações necessárias. Assim, o instrumento de pesquisa resultante se adequaria mel hor às especificidades do setor de telecomunicações e a uma realidade diferente da original, no caso, a brasileira.
Para a emersão dos indicadores, foram realizados grupos de foco, que consistem em uma técnica muito adequada à fase qualitativa. Foram sel ecionados grupos homogêneos em termos de faixa etária ou renda ou psicografia semelhante, abrangendo dois segmentos: estudantes universitários (três grupos) e estudantes do ensino médio (três grupos).

As entrevistas com os grupos de foco foram real izadas até o momento em que se observou uma convergência e se percebeu que os resul tados eram semel hantes e não se acrescentavam novas idéias. Com isso se obtiveram seis grupos de foco na fase qualitativa da pesquisa. 0 mediador - no caso o próprio pesquisador - conduziu as entrevistas utilizando-se de um roteiro com tópicos essenciais para a pesquisa. Para analisar os relatos dos entrevistados e alcançar os indicadores desejados foi utilizada a análise de conteúdo. Dentre as várias técnicas de análise de conteúdo, a análise temática se mostrou a mais adequada para 0 estudo, sendo utilizada a abordagem de Bardin (1977). Essa técnica consiste na contagem de um ou vários temas ou itens de significação, numa unidade de codificação previamente determinada.

Após uma primeira análise de identificação e classificação dos indicadores nos seus respectivos conceitos, prosseguiu-se com uma segunda análise, mais criteriosa, confrontando os resultados com a teoria. Em conseqüência, fez-se uma reclassificação dos indicadores, agrupando-os em seus respectivos construtos. Como resultado final dessa fase exploratória, obteve-se a primeira versão do instrumento de pesquisa, o qual foi submetido à apreciação de um especialista, doutor em marketing.

É importante afirmar que na análise das entrevistas com o grupo de foco foram observados aspectos que enunciaram al gumas mudanças no modelo. A maioria dos entrevistados (95,7\%) afirmou que avaliava a qualidade dos serviços de tel efonia comparando-o diretamente com as expectativas que tinha em relação a eles. Tendo em vista essa evidência, decidiu-se fazer a fusão de "expectativas" com "qual idade percebi da", como no estudo de Marchetti e Prado (2001), e seguindo a proposição de Brown, Churchill e Peter (1993) de que as medidas de percepções de qualidade fossem comparadas diretamente com as expectativas na hora de avaliar a qualidade percebida.

Outra observação importante obtida durante as entrevistas e em sua análise foi a não identificação de indicadores de reclamação. Ao falarem livremente, os entrevistados não evidenciaram a reclamação como um 
conceito importante. Por isso, o modelo sofreu algumas al terações após a análise da fase exploratória.

A análise originada dos relatos dos grupos de foco foi também submetida a uma comparação com alguns model os teóricos de satisfação. Verificou-se uma forte semelhança com o modelo aplicado por Marchetti e Prado (2001), o que levou à adoção desse modelo como base para a pesquisa deste artigo.

Feitas essas considerações, aplicou-se a primeira versão do instrumento de pesquisa a uma amostra selecionada, por conveniência, de 171 respondentes. 0 critério de definição do tamanho da amostra baseouse na recomendação de Hair et al. (1998) de um limite mínimo de cinco respondentes por parâmetro estimado. 0 objetivo da aplicação dessa versão do questionário foi o refinamento da escala, bem como a verificação da validade do instrumento para aplicação na amostra final. Seguindo as etapas propostas por Churchill (1979), essa foi a quarta etapa dentro desse modelo, a qual envolveu a avaliação da consistência interna pelo coeficiente alfa de Cronbach e a aplicação da análise fatorial exploratória.

$\mathrm{Na}$ análise da consistência interna dos 27 itens em seus respectivos construtos, observou-se que o coeficiente alfa de Cronbach de todos os construtos demonstrou confiabilidade aceitável, acima do mínimo recomendado por Hair et al. (1998). A análise do coeficiente alfa possibilitou o refinamento da escala, uma vez que foram apresentados itens que, se eliminados, aumentariam a confiabilidade (alfa). Com base nessa primeira análise, decidiu-se eliminar alguns itens que, se excluídos, aumentariam a confiabilidade da escala, pois o objetivo era atender ao princípio de parcimônia na elaboração de escalas.

Após a análise inicial, foi realizada a análise fatorial para verificar o agrupamento dos indicadores e sua respectiva carga fatorial. Analisando-se os valores, percebeu-se que alguns indicadores se agruparam nos fatores supostos. Outros indicadores supostamente pertencentes a construtos distintos se agruparam em um único fator, dificultando a análise, mesmo optando-se pela rotação não ortogonal (Oblimin), a qual é utilizada quando os itens são intercorrelacionados.

A partir dessa análise foram verificados os agrupamentos, e os indicadores foram submetidos a uma análise qualitativa apurada por dois especialistas do setor de telecomunicações, atuantes na área de pesquisa de mercado (validação de conteú do). A pós a combinação das duas técnicas (análise fatorial e validação de conteúdo), implementaram-se al terações semânticas e a retirada de itens. Buscando-se a parcimônia e a facilidade de compreensão da escala, as sugestões dos especialistas foram a modificação da escala de 10 para 5 pontos e a redução do número de questões em virtude da técnica de coleta de dados - sistema Computer Assisted Telephone Interview (CATI). Segundo eles, e também com base em Bailey (1992), essas mudanças auxiliariam a compreensão, por parte dos respondentes, do significado de cada rótulo da escala, e um número menor de questões facilitaria a aplicação por tel efone.

Como resultado da análise qualitativa, foi elaborado o instrumento final de pesquisa, contendo 18 questões referentes ao modelo (veja a Tabela 1). Embora a escala elaborada para a mensuração dos construtos do modelo original ACSI contenha 10 pontos, a comparação do modelo proposto com o ACSI não ficou prejudicada. Conforme a argumentação de Pasquali (1999), o número de pontos implementados nas escalas Likert parece al go irrelevante, pois esse número não afeta a consistência interna da escala Likert, nem a estabilidade teste-reteste, nem a validade concorrente e preditiva. Contudo, vale lembrar que a opção por uma escala de 5 pontos pode implicar a necessidade de amostras maiores para captar a variabilidade e minimizar o efeito do erro. Por isso, selecionou-se uma amostra maior para atender a esse objetivo. No presente artigo, a escala Likert utilizada foi tratada como intervalar para efeitos de testes estatísticos, como na maioria dos trabal hos que utilizam a metodologia survey.

\section{Fase quantitativa/ descritiva}

A segunda fase da pesquisa consistiu em uma pesquisa do tipo survey, correspondente à etapa 5 do modelo de Churchill (1979). Para o cálculo da amostra, foi observada a recomendação de Hair et al. (1998), que indicam um mínimo de cinco respondentes por parâmetro estimado. Assim, a amostra por operadora, perto de 200, atendeu a esse critério de tamanho mínimo para a amostra ( 5 x 38 parâmetros $=190$ ). Apesar de a quantia mínima estar dentro desse critério, optou-se por não realizar os testes por operadora e obter maior confiabilidade dos resultados com uma amostra maior. Então, a amostra para os testes do modelo foi considerada como um todo, agregando as três operadoras, num total de 606 respondentes. Considerando os critérios para cálculo amostral expostos por Samara e Barros (2002), uma amostra mínima de 384 casos seria necessária para atingir uma proporção de $95 \%$ de margem de segurança, com $5 \%$ de erro, para 
uma população de 4,5 milhões de usuários em Minas Gerais. Os erros de medida foram avaliados para a amostra total.

Devido à estrutura e aos recursos disponibilizados pela empresa parceira na pesquisa, utilizou-se como técnica de coleta de dados o sistema CATI. É importante observar que essa mesma técnica de coleta de dados foi implementada pelos pesquisadores proponentes do modelo ACSI, no qual o presente estudo se baseia. Dessa forma, a empresa colaboradora na pesquisa prontificou-se a organizar a coleta de dados, alocando pessoal, selecionando al eatoriamente os respondentes e garantindo todos os recursos necessários para a realização dessa fase quantitativa. Para isso, seis atendentes do setor de pesquisa ficaram responsáveis por fazer as ligações, entrevistar os clientes e preencher os formulários de pesquisa, de acordo com a técnica CATI. Além disso, informaram o objetivo da pesquisa e asseguraram 0 anonimato dos respondentes. Todo o procedimento foi acompanhado por dois supervisores, em dois turnos (matutino e vespertino).

Tabela 1 - Análise descritiva da amostra.

\begin{tabular}{l} 
DESCRIÇÃO DO INDICADOR \\
1 Qualidade e facilidade das ligaç̃oes \\
2 Cobertura suficiente para atender as necessidades dos clientes \\
\hline 3 Atendimento telefônico (central de atendimento) \\
\hline 4 Atendimento nas lojas próprias e agentes credenciados \\
\hline 5 Modelos de aparelho celular disponibilizados compatíveis... \\
\hline 6 Serviço livre de falhas e deficiências \\
\hline 7 Justiça e transparência nos preços praticados e cobranças \\
\hline 8 Qualidade dos serviços compatível com o preço pago \\
\hline 9 Qualidade do atendimento telefônico e lojas compatível ... \\
\hline 10 Serviços adicionais compatíveis com o preço pago \\
\hline 11 A operadora oferece facilidades que o fazem ganhar tempo \\
\hline 12 Os serviços estão sempre disponíveis na hora que você precisa \\
\hline 13 Satisfação global \\
\hline 14 Desconfirmação de expectativas \\
\hline 15 Desempenho versus produto/ serviço ideal \\
\hline 16 Se pudesse manter o número de celular, trocaria de operadora \\
\hline 17 Se as outras oferecessem o benefício de falar $20 \%$ a mais ... \\
\hline 18 Se os preços dos serviços das outras fossem $10 \%$ mais baratos \\
\hline
\end{tabular}

É importante salientar que um pré-teste do questionário foi realizado utilizando o sistema CATI, a fim de verificar inconsistências nas questões e o tempo de resposta antes da aplicação final do questionário. Todas as entrevistas foram realizadas em três dias, e alcançou-se uma amostra de 606 casos válidos.

A etapa de coleta de dados ocorreu nos dias 17 e 20 de outubro de 2003 e no dia 3 de novembro de 2003, conforme disponibilidade da empresa col aboradora na pesquisa. Os dados relativos à pesquisa survey receberam tratamento estatístico por meio dos softwares SPSS 11.0, Excel e AMOS 4.0, que foram utilizados para a análise dos dados. A análise descritiva dos dados contendo as respostas médias das variáveis e o desvio padrão consta da Tabela 1.

\section{ANÁLISE DOS DADOS}

Com base na análise inicial dos dados, identificaramse quatro outliers multivariados, que foram retirados

\begin{tabular}{|l|l|r|}
\hline \multicolumn{1}{|c|}{ ESCALA } & MÉDIA & $\begin{array}{r}\text { DESVIO } \\
\text { PADRÃO }\end{array}$ \\
\hline 1 Muito pior q. o esperado até & 3,546 & 0,996 \\
\hline 5 Muito melhor... & 3,045 & 1,175 \\
\hline & 3,487 & 1,165 \\
\hline & 3,518 & 1,070 \\
\hline & 3,942 & 0,970 \\
\hline & 3,381 & 1,056 \\
\hline & 3,053 & 1,156 \\
\hline 1 Discorda totalmente até & 3,244 & 1,225 \\
\hline 5 Concorda totalmente & 3,495 & 1,171 \\
\hline & 3,597 & 1,275 \\
\hline 1 Discorda totalmente & 3,800 & 1,208 \\
\hline 5 Concorda totalmente & 3,667 & 1,344 \\
\hline 1 M.insatisfeito/ 5 M. satisfeito & 3,691 & 0,892 \\
\hline 1 M. abaixo/ 5 M. acima expect. & 3,045 & 0,829 \\
\hline 1 Muito longe do ideal até & 3,637 & 0,931 \\
\hline 5 Muito perto... & 3,378 & 1,183 \\
\hline 1 Certamente trocaria até & 2,673 & 1,214 \\
\hline 5 Certamente não trocaria & 2,736 & 1,168 \\
\hline
\end{tabular}


da análise para não comprometer os resultados. Portanto, a amostra final correspondeu a 602 casos válidos. Com relação à análise de pressupostos das técnicas multivariadas, a normalidade dos dados não foi verificada. Contudo, optou-se por aplicar as técnicas de anál ise multivariada sem utilizar transformações, e utilizar métodos de estimação mais robustos à nãonormalidade para a análise multivariada dos dados. Além disso, considerou-se o argumento dos autores Hair et al. (1998) e Gujarati (2000) de que os efeitos negativos da não-normalidade tendem a ser menos danosos em amostras grandes, como é o caso da amostra em estudo (602 casos válidos).

As variáveis do modelo apresentaram correlações entre si, indicando haver uma relação linear positiva moderada entre elas. A homocedasticidade não foi observada. Os pré-requisitos para se realizar a Análise Fatorial Exploratória (AFE) foram apurados, o que indicou a aplicação da técnica, pois KMO foi mai or que 0 mínimo aceitável $(0,70)$ e o teste Bartlett $p<0,05$.

Em relação à validação do instrumento de pesquisa, os resultados da AFE indicaram uma reconfiguração do modelo hipotético proposto (veja a Tabela 2). Isso em virtude de o construto qualidade percebida apre- sentar duas dimensões (sendo os indicadores desse construto agrupados em dois fatores) e os indicadores do construto conveniência não se agruparem em uma dimensão.

Antes de qual quer al teração no modelo, a escal a com o formato indicado pela AFE foi submetida ao teste de confiabilidade do instrumento de pesquisa pelo coeficiente al pha de Cronbach. N esse ponto, os coeficientes calculados para cada construto apresentaram valores acima do mínimo $(0,60)$, atestando a confiabilidade da escala. Somente a escala do construto conveniência se mostrou com baixa confiabilidade (alpha $=0,2858$ ).

Como resultado da análise fatorial exploratória e da confiabilidade da escala pelo coeficiente alpha de Cronbach, o modelo inicialmente proposto passou por al gumas alterações, sendo o construto qualidade subdividido em duas dimensões - qualidade de acesso e qualidade geral -, e o construto conveniência retirado da análise por não apresentar agrupamento em um fator, pelo fato de a confiabilidade medida pelo alpha estar muito aquém do aceitável.

Para avaliar com mais profundidade a confiabilidade da escala, foram realizados os testes de confiabilidade composta e variância extraída (veja a Tabela 3).

Tabela 2 - Matriz das cargas fatoriais.

\begin{tabular}{|c|c|c|c|c|c|}
\hline \multicolumn{6}{|c|}{ FATORES } \\
\hline & 1 & 2 & 3 & 4 & 5 \\
\hline QUAL6 & 0,547 & & & & \\
\hline QUAL3 & 0,511 & & & & \\
\hline QUAL4 & 0,510 & & & & \\
\hline QUAL5 & 0,404 & & & & \\
\hline QUAL7 & 0,344 & & & & \\
\hline LEAL17 & & 0,884 & & & \\
\hline LEAL18 & & 0,717 & & & \\
\hline QUAL1 & & & $-0,620$ & & \\
\hline QUAL2 & & & $-0,575$ & & \\
\hline CONV12 & & & $-0,473$ & & \\
\hline VALOR9 & & & & 0,670 & \\
\hline VALOR8 & & & $-0,321$ & 0,454 & \\
\hline VALOR10 & & & & 0,450 & \\
\hline \multicolumn{6}{|l|}{ CONV11 } \\
\hline SATIS15 & & & & & $-0,676$ \\
\hline SATS13 & & & & & $-0,601$ \\
\hline SATS14 & & & & & $-0,596$ \\
\hline LEAL16 & 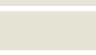 & 0,352 & & & $-0,393$ \\
\hline
\end{tabular}


A confiabilidade composta e a variância extraída são apuradas para cada construto, utilizando os pesos padronizados resultantes da AFC e dos erros de mensuração dos indicadores ( Hair et al. 1998). Esses autores consideram a confiabilidade aceitável quando denotada por valores iguais ou mai ores que 0,70 para a confiabilidade composta e iguais ou mai ores que 0,50 para a variância extraída. Os resultados obtidos atestaram a confiabilidade composta para todos os construtos em análise. Porém, os valores de variância extraída dos construtos ficaram um pouco abaixo do limite mínimo de 0,50 , e somente o construto satisfação apresentou variância extraída de 0,70 . Isso indica um ponto de atenção maior para o pesquisador no cálculo da validade convergente.

Para cada construto verificou-se a validade convergente, que indica, segundo Malhotra (2001), até que ponto a escala se correlaciona positivamente com outras medidas do mesmo construto. A verificação da validade convergente foi realizada pelo exame da significância estatística dos parâmetros estimados pela análise fatorial confirmatória (AFC), observando os seus respectivos t-values (Perin, 2001). Segundo o exame da significância estatística, considera-se a validade convergente aceitável quando cada uma das cargas fatoriais (parâmetros estimados) relativas aos indicadores dos construtos é estatisticamente significativa, ou seja, 0 valor $p$ é menor que 0,05 ou o t-value é maior ou igual a 1,96. Cinco modelos de análise fatorial foram avaliados, um por construto. Verificou-se que as cargas fatoriais estimadas para cada construto foram significativas em um nível de $5 \%(p<0,05)$. Isso quer dizer que os indicadores convergem para o seu respectivo construto com carga fatorial estatisticamente significante.

A validade discriminante refere-se ao grau em que uma medida não se correlaciona com outros construtos, dos quais se supõe que difira. Assim, a validade discriminante dos construtos do modelo proposto (qualidade percebida - duas dimensões, valor percebido, satisfação e lealdade) foi avaliada par a par com o objetivo de avaliar se as variáveis latentes eram nãocorrelacionadas e, portanto, discriminantes. Esse procedimento pode ser feito por meio da diferença de quiquadrado, conforme Gosling (2001), abalizada em Jöreskog e Sörbom (1996), e M oorman e M iner (1998). Os pares de construtos foram testados com perfeita correlação, ou seja, imputan do uma correlação igual a 1; em seguida, forçava-se a não-correlação com o valor zero. A diferença considerada significativa entre os qui-quadrados dos model os deve ser maior que 3,84 . Assim, utilizando esse critério apontado pelos autores, foi apurada a diferença entre os qui-quadrados dos pares de construtos do model o proposto. Conforme a Tabela 4, observou-se que a diferença de qui-quadrados em todos os pares de construtos foi significativa, sendo a estatística qui-quadrado dos modelos não correlacionados menor, ou seja, apresentando um ajustamento melhor. Em outras palavras, isso indica que os construtos são não-correlacionados, o que atesta a validade discriminante dos construtos.

\section{Análise do modelo proposto}

Após a análise de validação do instrumento de pesquisa, bem como da avaliação das premissas requeridas pela análise multivariada, partiu-se para a aplicação da técnica de model agem de equações estruturais, tendo por finalidade a verificação das hipóteses concernentes ao modelo proposto. Todas as hipóteses foram submetidas a testes, com exceção das hipóteses referentes ao construto conveniência.

Para ser implementada a técnica de equações estruturais, Hair et al. (1998) apontam sete etapas pertinentes: 1) desenvolvimento de um modelo com base na teoria; 2) construção do diagrama de caminhos; 3 ) conversão do diagrama de caminhos; 4) seleção da matriz de entrada de dados e estimação do modelo; 5) avaliação da identificação do model o; 6) avaliação das estimativas do modelo e do goodness-of-fit; 7) interpretação e modificação do modelo.

A realização da primeira, segunda e terceira etapas

Tabela 3 - Confiabilidade composta e variância extraída.

\begin{tabular}{|l|c|c|}
\hline \multicolumn{1}{|c|}{ CONSTRUTO } & VALOR DA CONFIABILIDADE COM POSTA & VARIÂNCIA EXTRAíDA \\
\hline Qualidade geral & 0,76 & 0,40 \\
\hline Qualidade acesso & não calculada (no insuficiente de itens) & Não calculada \\
\hline Valor & 0,61 & 0,34 \\
\hline Satisfação & 0,87 & 0,70 \\
\hline Lealdade & 0,70 & 0,45 \\
\hline
\end{tabular}


implicou a construção do modelo estrutural e de mensuração. Em relação à sel eção da matriz de entrada de dados, decidiu-se pela utilização da matriz de covariância, que - de acordo com Hair et al. (1998) - é mais adequada à validação de um modelo contemplando rel acionamentos entre antecedentes e conseqü entes de determinado construto, no caso, a satisfação.

0 método de estimação sel ecionado para os testes de equações estruturais foi o Generalized Least Squares (GLS), o qual é um método mais robusto à não-normalidade dos dados. Esse método também foi implementado por Gonçalves Filho (2001) devido ao não alcance da normalidade multivariada. Selecionada a matriz de entrada de dados, bem como o método de estimação, os resultados puderam ser al cançados e analisados, dando prosseguimento às demais etapas propostas por Hair et al.

0 modelo integrado que emergiu da AFE foi submetido a testes considerando a base de dados geral (todas as operadoras) sem os outliers multivariados, num total de 602 casos válidos. Tal modelo é mostrado na Figura 3.
Analisando-se as medidas de ajustamento do modelo, observou-se em um primeiro momento o teste de qui-quadrado, dado pelo valor $\mathrm{p}$. Para o modelo proposto, 0 valor $p=0,00$ foi inferior ao mínimo exigido $(p=0,05)$. Assim, a hipótese nula do teste, de que a matriz de dados observada é igual à estimada pelo modelo, foi rejeitada. No entanto, como a amostra total (602) é considerada grande, e segundo Hair et al. (1998) o teste de qui-quadrado é mais sensível e menos confiável para amostras maiores que 200 casos, os autores recomendam que o pesquisador complemente sua análise com outras medidas de ajuste para a verificação da val idade do modelo. Dessa forma, no trabalho em questão, outras medidas de ajustamento foram verificadas.

Quanto às outras medidas, Hair et al. mostram que os principais indicadores de ajuste - GFI, AGFI, CFI, $\mathrm{NFI}, \mathrm{TLI}$ - possuem bom desempenho, com valores iguais ou acima de 0,90. Observando esse critério, 0 modelo proposto da pesquisa apresentou um nível moderado de ajuste. A verificação das medidas permi-

Figura 3 - Modelo de mensuração.

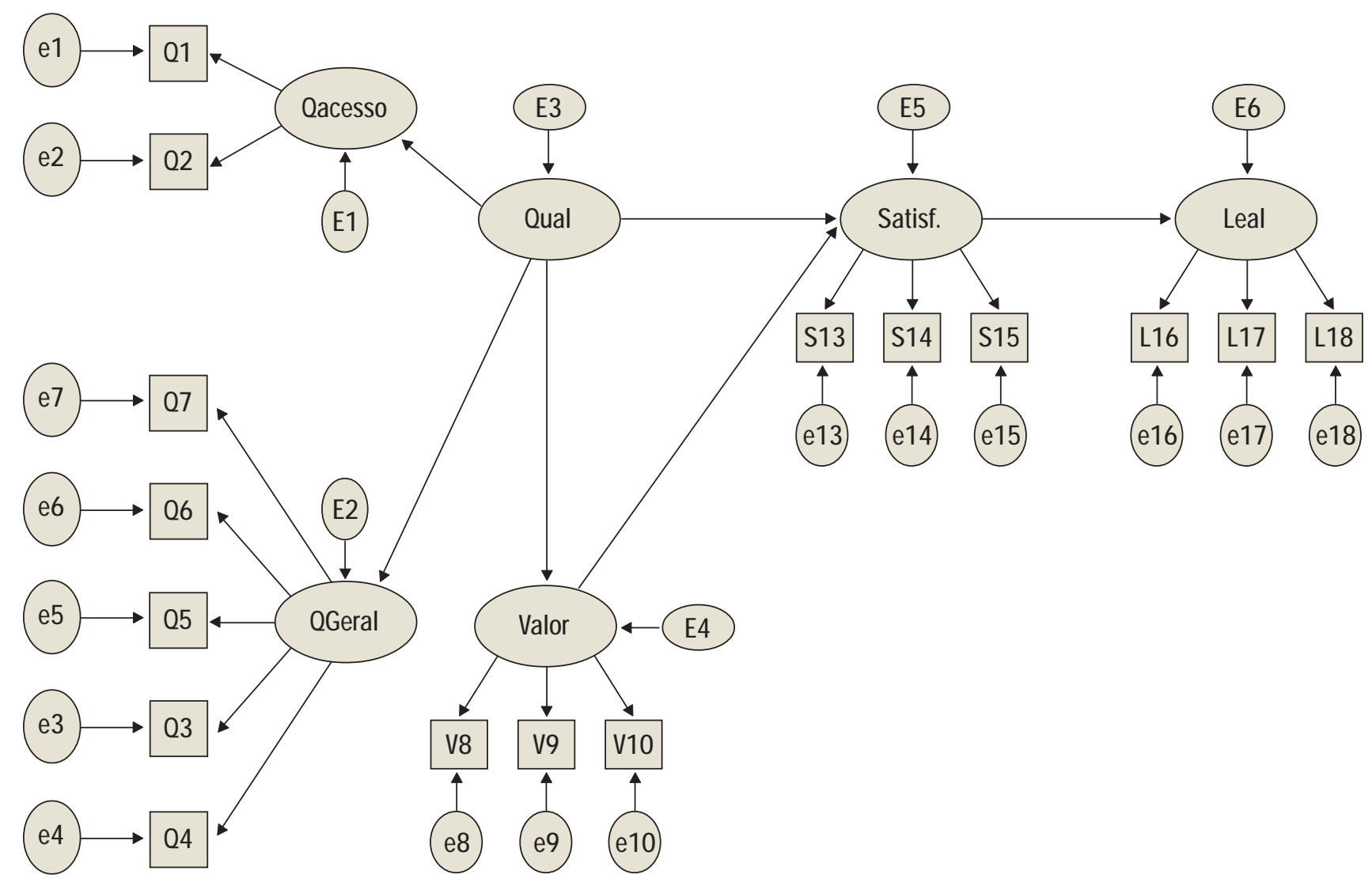


te dizer que 0 ajuste absoluto ocorreu ao considerar que o modelo apresentou $\mathrm{GFI}=0,945$ e AGFI =0,923, apesar de 0 valor $p$ ser menor que 0,05 (veja a Tabela 5). 0 valor de RMSEA $=0,053$ favoreceu a aceitação do modelo porque é menor que 0,08 . 0 ajustamento parcimonioso também foi verificado, pois o qui-quadrado normalizado $\left(x^{2} / g l\right)$ apresentou valor de 2,17, abaixo do limite superior, de 5 . Contudo, as medidas de ajuste incremental do modelo apresentaram valores abaixo do recomendável, $\mathrm{NFI}=0,615, \mathrm{CFI}=0,706$ e $T L I=0,640$. Isso pode significar a necessidade de melhorias no modelo proposto. Este não excedeu 0 modelo nulo, que é realista, embora mais simples, e que deve ser excedido pelos demais modelos por serem considerados melhores. U ma das razões para 0 nível de ajuste encontrado foi o fato de não ter sido atingida a unidimensional idade para o construto qualidade percebida.

Assim, com base nos índices de ajuste, o modelo proposto da pesquisa apresenta um nível moderado de ajustamento global. Esse resultado, porém, favorece a avaliação do ajustamento dos aspectos de mensuração e permite prosseguir com 0 teste das hipóteses do modelo (Huertas e Urdan, 2000).

De acordo com os resultados apresentados para 0 modelo proposto, todas as relações entre os construtos mostraram-se estatisticamente significativas, pois os pesos $(\beta)$ das relações apresentaram $p<0,05$ (veja a Tabela 6). Dessa forma, pode-se interpretar que qualidade apresentou um forte vínculo com valor $(\beta=$ $0,86)$, isto é, quanto maior a percepção de qualidade, maior o valor percebido. 0 coeficiente $\beta$ entre os construtos qualidade e satisfação apresentou o valor de 0,624 , indicando um relacionamento conseqüencial positivo de moderado a al to. Q uanto ao relacionamento entre valor e satisfação, o peso foi menor $(0,295)$, indicando uma relação positiva, com o construto valor afetando a variável latente satisfação com uma intensidade menor.

0 vínculo entre o par de construtos satisfação e leal dade mostrou-se forte, com um $\beta$ de 0,776 . I sso significa que o relacionamento entre os construtos é direto e positivo, ou seja, quanto maior a satisfação do cliente, maior é a sua leal dade à operadora.

Com respeito ao poder de explicação dos construtos da cadeia nomológica de relações, percebeu-se que: 1) a variância de Q acesso é explicada em $85 \%$ pela variável latente qualidade; 2 ) a variância de Q geral é explicada em $73,4 \%$, pela variável latente qualidade; 3) a variância de val or percebido é explicada em $73,9 \%$ pelo construto qualidade; 4) a variância de satisfação é explicada em $79,4 \%$ pelos construtos valor e qualidade; 5) a variância de lealdade é explicada em 60,3\% pela satisfação do cliente.

\section{CONSIDERAÇÕES FINAIS E CONCLUSÕES}

Observou-se que o modelo proposto sugerido pela análise fatorial exploratória apresentou um nível moderado de ajuste, pela AFC. A análise das hipóteses permitiu dizer que os antecedentes de satisfação ( qualidade percebida e valor percebido) afetam a satisfação, sendo, pela análise dos $\beta$ 's padronizados do modelo, a qualidade percebida mais importante que 0 valor percebido, respectivamente 0,624 e 0,295 . A relação conseqüencial de que a satisfação do consumidor afeta sua lealdade foi também verificada, como se

Tabela 4 - Diferenças de qui-quadrados entre os pares de construtos.

\begin{tabular}{|c|c|c|c|}
\hline PAR DE CONSTRUTOS & CORR = $1 \quad$ QUI-QUADRADO & CORR $=0 \quad$ QUI-QUADRADO & DIFERENÇA QUI-QUADRADO \\
\hline Qq_satisf & 267,764 & 173,648 & 94,116 \\
\hline Qg_leal & 436,304 & 134,558 & 301,746 \\
\hline Qg_valor & 363,071 & 192,264 & 170,807 \\
\hline Leal_satisf & 335,888 & 166,161 & 169,727 \\
\hline Leal_valor & 154,839 & 140,432 & 14,407 \\
\hline Satisf_valor & 335,893 & 157,822 & 178,071 \\
\hline
\end{tabular}

Tabela 5 - Resultados da análise fatorial confirmatória para 0 modelo.

\begin{tabular}{|c|c|c|c|c|c|c|c|c|}
\hline$x^{2}$ & GFI & AGFI & NFI & $\mathrm{CFI}$ & TLI & $X^{2} / g l$ & RMSEA & P-VALOR \\
\hline 266,276 & 0,945 & 0,923 & 0,615 & 0,706 & 0,640 & 2,717 & 0,053 & 0,00 \\
\hline
\end{tabular}


constatou também no trabalho de $M$ archetti e Prado (2001), Gonçalves Filho, Guerra e Moura (2003), e Huertas e Urdan (2000).

É importante destacar um dos objetivos específicos deste estudo, que foi o cálculo dos índices ACSI de satisfação, a fim de verificar a avaliação dos clientes quanto às operadoras em questão. Tais índices foram obtidos pela aplicação da mesma fórmula utilizada por Fornell et al. (1996), uma vez que o modelo proposto pode ser comparado com o original porque foram mantidos os mesmos três indicadores de satisfação. Assim, o índice foi obtido pela seguinte fórmula:

$$
S G C=\frac{\sum p i \cdot \overline{x i}-\sum p j \cdot \operatorname{Min}(x i)}{\sum p i \cdot \operatorname{Mar}(x i)-\sum p i \cdot \operatorname{Min}(x i)} \times 100
$$

onde:

pi, pesos calculados para os indicadores da satisfação global.

$\overline{x i}$, valor médio obtido para cada indicador de satisfação.

Min(xi), valor mínimo da escala correspondente. Max(xi), valor máximo da escala correspondente. SGC, índice de satisfação global do consumidor.

Utilizando-se a fórmula, os índices ACSI de satisfação gerados pelo presente estudo foram os seguintes: O peradora $X(65,30 \%)$, Operadora $Y(64,70 \%)$ e Operadora Z (56,07\%). Contudo, esses resultados não distam muito dos verificados no setor de telecomunicações nos Estados Unidos, de onde provém o modelo. $\mathrm{N}$ a última referência de 2004, o índice global do setor foi de $71 \%$. Nos outros anos, os indicadores foram: $75 \%$ em 1997; $74 \%$ em 1998; 73\% em 1999; $72 \%$ em 2000; 70\% em 2001; 71\% em 2002; e 72\% em 2003 (Fornell et al., 2005).

A análise dos índices possibilita dizer que os resultados alcançados em relação às operadoras mineiras

Tabela 6 - Resumo das relações entre os construtos

\begin{tabular}{l|c|c}
\hline \multicolumn{1}{|c|}{ RELAÇÃO } & VALORES $(\beta)$ & P-VALOR \\
\hline Qualidade $\rightarrow$ Qualidade acesso & 0,922 & 0,000 \\
\hline Qualidade $\rightarrow$ Qualidade geral & 0,857 & 0,000 \\
\hline Qualidade $\rightarrow$ Valor & 0,860 & 0,000 \\
\hline Qualidade $\rightarrow$ Satisfação & 0,624 & $-*$ \\
\hline Valor $\rightarrow$ Satisfação & 0,295 & 0,039 \\
\hline Satisfação $\rightarrow$ Lealdade & 0,776 & 0,000 \\
\hline Nota: * Não calculado - peso não padronizado foi fixado em 1 pelo software & &
\end{tabular}

apresentam índices de satisfação ainda aquém do seu diz respeito à percepção dos usuários. Provavel mente, por se tratar de um setor que se desenvolveu recentemente no Brasil, que está em franca expansão e vem passando por profundas transformações, é possível considerar um grande potencial de melhoria na avaliação de seus clientes.

A principal contribuição deste trabal ho tanto numa perspectiva prática quanto teórica reside na metodoto de pesquisa empregando técnicas de abordagem qualitativa (grupos de foco), grupo de discussão de especialistas com acadêmicos e práticos e técnicas de abordagem quantitativas, culminou num rico trabaIho de pesquisa, não obstante as limitações que ocorreram durante a execução dessa pesquisa.

A integração entre o conhecimento prático e o teórico, buscando-se ouvir as opiniões tanto dos praticantes de pesquisa da Administração quanto dos acadêmicos, proporcionou grandes ganhos no conhecimento sobre o tema. Ademais, apontam-se como contribuições deste trabalho: a) o reforço da teoria que relaciona o construto satisfação com seus principais antecedentes e conseqüentes; b) a recomendação gerencial de que investimentos nos antecedentes qualidade e valor se reproduzem em efeitos positivos na satisfação do consumidor e conseqüentemente na leal dade à empresa.

No que se refere a saber até que ponto o modelo de satisfação ACSI modificado é válido para o setor de tel efonia móvel de M inas Gerais, constatou-se um nível de ajuste moderado para o modelo proposto com base na análise fatorial exploratória. Para as hipóteses contidas no model o gerado, todas el as foram apoiadas pelos testes, considerando-se a base de dados geral. As seguintes hipóteses foram apoiadas pela análise de dados: Qualidade $\rightarrow$ Satisfação; Qualidade $\rightarrow$ Valor Percebido; Valor Percebido $\rightarrow$ Satisfação; Satisfação $\rightarrow$ potencial, havendo oportunidades de melhoria no que logia adotada. 0 esforço de adaptação do instrumen-

Nota: * Não calculado - peso não padronizado foi fixado em 1 pelo software. 
Leal dade (veja o Quadro 1).

Um importante aspecto observado no processo de pesquisa foram as alterações emanadas do trabalho empírico para a teoria proposta, levando o pesquisador, em todos os momentos, a rever técnicas, procedimentos e hipóteses a serem avaliadas. 0 emprego de métodos qualitativos no presente estudo foi uma tentativa de aproximação da realidade dos consumidores da região pesquisada.

As limitações da pesquisa se referem à aplicação das técnicas para a coleta de dados tanto para a fase qualitativa quanto para a quantitativa, além das restrições de tempo e recursos financeiros. A escol ha de obtenção de informações por grupos de foco, na fase inicial da pesquisa, implica restrições. Conforme aponta M al hotra (2001), a natureza não-estruturada das respostas dos participantes pode dificultar a codificação, a análise e a interpretação dos resultados, além da possibilidade de não serem representativos da população geral, porém essa técnica apresenta importantes vantagens.

Para a fase quantitativa, outras limitações foram a utilização do método survey, bem como seu modo de aplicação, o sistema CATI. A desvantagem encontrada no método survey é que os entrevistados podem não fornecer a informação correta ou não compreender as perguntas, relutar em responder a questões delicadas ou pessoais ( $M$ alhotra, 2001). Além disso, o modo de aplicação de survey por tel efone e com o auxílio do computador pode apresentar um nível moderado de tendenciosidade do entrevistador, resultando em viés e influenciando na qualidade dos dados.

Considerando os construtos propostos, verificouse que, para o construto denominado conveniência, não foi possível levantar indicadores que mensurassem tal conceito. Nesse ponto, fica a sugestão, para pesquisas futuras, de tentar al cançar esses indicadores ou avaliar a sua importância como parte do modelo de satisfação. Ainda são necessários mais esforços para o alcance de indicadores do construto qualidade, a fim

Quadro 1 - Teste de hipóteses.

\begin{tabular}{l|l|}
\hline HIPÓTESES ALTERNATIVAS & RESULTADO \\
\hline Qualidade $\rightarrow$ Qualidade acesso & Apoiada \\
\hline Qualidade $\rightarrow$ Qualidade geral & Apoiada \\
\hline Qualidade $\rightarrow$ Satisfação & Apoiada \\
\hline Qualidade $\rightarrow$ Valor & Apoiada \\
\hline Valor $\rightarrow$ Satisfação & Apoiada \\
\hline Satisfação $\rightarrow$ Lealdade & Apoiada \\
\hline
\end{tabular}

de torná-lo unidimensional.

A generalização dos resultados deste estudo para o Brasil fica comprometida, uma vez que sua aplicação restringiu-se ao estado de Minas Gerais. Sugere-se a ampliação desta pesquisa contemplando uma amostra dos clientes de todas as operadoras de tel efonia móvel do Brasil, inclusive avaliando os vários segmentos de clientes com plano individual e/ou corporativo, utilizando-se como critérios a intensidade de uso do serviço, o tempo de experiência com o serviço e outros.

Iniciativas no sentido de ampliar os estudos de aplicação do modelo ACSI no setor de telefonia móvel brasileiro seriam de grande valia para o al cance de um modelo de satisfação ainda mais adequado, de modo que possa refletir o comportamento do cliente desse setor, no que se refere à sua satisfação e ao relacionamento com outras variáveis importantes.

\section{REFERÊNCIAS BIBLIOGRÁFICAS}

ANDERSON, E. W.; FORNELL, C. Foundations of the American Customer Satisfaction Index. Total Quality Management, v. 11, n. 7, 2000.

ANDERSON, E. W.; FORNELL, C.; LEHMAN, D. R. Customer satisfaction, market share, and profitability: findings from Sweden. Journal of M arketing, v. 58, p. 53-66, 1994.

BAILEY, K. D. M ethods of Social Research. New York: The Free Press, 1992. BARDIN, L. Análise de conteúdo. Lisboa: Edições 70, 1977.

BERRY, L. L.; SEIDERS, K.; GREWAL, D. Understanding service convenience. Journal of Marketing, v. 66, p. 1-17, 2002.

BOULDING, W. et al. A dynamic process model of service quality: from expectations to behavioral intentions. Journal of M arketing Research, v. 30, p. 7-27, 1993.

BROW N, L. G. Convenience in services marketing. The Journal of Services Marketing, v. 4, n. 1, 1990.

BROWN, T. J.; CHURCHILL JR., G. A.; PETER, J. P. Research note: improving the measurement of service quality. Journal of Retailing, v. 69, p. 127-139, 1993.

CARDOZO, R. N. An experimental study of customer effort, expectation, and satisfaction. Journal of M arketing Research, v. 2, p. 244-249, 1965.

CHURCHILL JR., G. A. A paradigm for developing better measures of marketing constructs. Journal of Marketing Research, v. 16, n. 1, p. 63-73, 1979.

CHURCHILL JR., G. A.; SURPRENANT, C. An investigation into the determinants of customer satisfaction. Journal of M arketing Research, v. 19, p. 491-504, 1982.

FORNELL, C. A National customer satisfaction barometer: the Swedish experience. Journal of Marketing, v. 56, p. 6-21, 1992. 
FORNELL, C. et al. American Customer Satisfaction Index. Methodology Report. Ann Arbor, National Quality Research Center, 1995.

FORNELL, C. et al. The American Customer Satisfaction Index: nature, purpose, and findings. Journal of Marketing, v. 60, p.7-18, 1996.

FORNELL, C.; ITTNER, C. D.; LARCKER, D. F. The Valuation Consequences of Customer Satisfaction across Industries. Working Paper. Ann Arbor, National Quality Research Center, 1996.

FORNELL, C. et al. First Quarter Scores. Disponível em: < http:// www.theacsi.org/first_quarter.htm>. Acesso em: 26 fev. 2005.

GON ÇALVES FILHO, C. 0 impacto da gestão do conhecimento de marketing na inovação e vantagem competitiva de novos produtos. Tese (Doutorado em Administração). Cepead, UFM G, Belo Horizonte, 2001.

GONÇALVES FILHO, C.; GUERRA, R. S.; MOURA, A. Mensuração de satisfação, qualidade, leal dade, valor e expectativa em instituições de ensino superior: um estudo do modelo ACSI através de equações estruturais. In: ENCONTRO NACIONAL DA ASSOCIAÇÃO NACIONAL DOS PROGRAMAS DE PÓS-GRADUAÇÃO E PESQUISA EM ADMINISTRAÇÃO, 27., 2003, Atibaia. Anais. Atibaia: AN PAD, 2003.

GOSLING, M. Estratégias de relacionamento no setor bancário brasileiro: um estudo empírico. Dissertação (M estrado em Administração). Cepead, UFMG, Belo Horizonte, 2001.

GUJARATI, D. N. Econometria básica. São Paulo: Makron Books, 2000.

HAIR JR. et al. Multivariate Data Analysis. Englewood Cliffs, NJ: Prentice Hall, 1998.

HUERTAS, M. K. Z., URDAN, A. T. Satisfação do cliente com serviços de assistência técnica automobilística e lealdade dele ao fabricante do veícuIo. In: ENCONTRO NACIONAL DA ASSOCIAÇÃO NACIONAL DOS PROGRAMAS DE PÓS-GRADUAÇÃO E PESQUISA EM ADMINISTRAÇÃO, 24., 2000. Florianópolis. Anais. Florianópolis: AN PAD, 2000.

JÖRESKOG, K.; SÖRBOM, D. LISREL 8: U ser's Reference Guide. Chicago: SSI, 1996.

MALHOTRA, N. K. Pesquisa de marketing. Porto Alegre: Bookman, 2001.
MARCHETTI, R.; PRADO, P. Avaliação da satisfação do consumidor utilizando o PLS: um modelo aplicado ao setor elétrico brasileiro. In: ENCONTRO NACIONAL DA ASSOCIAÇÃO NACIONAL DOS PROGRAMAS DE PÓS-GRADUAÇÃO E PESQUISA EM ADMINISTRAÇÃO, 25. 2001. Campinas. Anais. Campinas: AN PAD, 2001.

MOORMAN, C.; MINER, A. S. The convergence of planning and execution: improvisation in new product development. Journal of Marketing. v. $62, n$. 3, 1998.

OLIVER, R. L. Satisfaction: A Behavioral Perspective on the Consumer. Boston: Irwin/ McGraw-Hill, 1997.

OLIVEIRA, R. Telecomunicação: a revolução pós-privatização. Banas Q ualidade, São Paulo, v. 102, p. 36-47, 2000.

PASQUALI, L. Instrumentos psicológicos: manual prático de elaboração. Brasília: LabPAM/ IBAPP, 1999.

PERIN, M. G. A relação entre orientação para o mercado, aprendizagem organizacional e performance. Tese (Doutorado em Administração). PPGA, UFRGS, Porto Alegre, 2001.

REICHHELD, F. F.; SASSER, W. E. Zero defections: quality comes to services. Harvard Business Review, v. 68, p. 105-11, sep.-oct. 1990.

ROSSI, C. A; SLONGO, L. A. Pesquisa de satisfação de clientes: o estadoda-arte e proposição de um método brasileiro. In: ENCONTRO NACIONAL DA ASSOCIAÇÃO NACIONAL DOS PROGRAMAS DE PÓS-GRADUAÇÃO E PESQUISA EM ADMINISTRAÇÃO, 21., 1997. Angra dos Reis. Anais. Angra dos Reis: AN PAD, 1997.

SAMARA, B. S.; BARROS, J. C. Pesquisa de marketing: conceitos emetodologia. São Paulo: Prentice Hall, 2002.

URDAN, A. T.; RODRIGUES, A. R. 0 modelo do índice de satisfação do cliente norte-americano: um exame inicial no Brasil com equações estruturais. In: EN CONTRO NACIONAL DA ASSO CIAÇÃO NACIONAL DOS PROGRAMAS DE PÓS-GRADUAÇÃO E PESQUISA EM ADMINISTRAÇÃO, 22., 1998. Foz do Iguaçu. Anais. Foz do Iguaçu: AN PAD, 1998.

\section{Artigo recebido em 23.08.2004. Aprovado em 18.07.2005.}

\section{Andréia Cássia de Moura}

Professora da Faculdade Batista de Minas Gerais e Faculdade Estácio de Sá. M estre em Administração pela UFMG-CEPEAD.

Interesses de pesquisa nas áreas de novas tecnologias gerenciais, mercadologia e administração estratégica.

E-mail: acassiabh@yahoo.com.br

Endereço: Rua Bueno do Prado, 222, Bairro João Pinheiro, Belo Horizonte - M G, 30530-430.

\section{C arlos Alberto G onçalves}

Professor da UFM G-FACE. Doutor em Administração pela USP.

Interesses de pesquisa nas áreas de novas tecnologias gerenciais, mercadologia e administração estratégica.

E-mail: carlos@face.ufmg.br

Endereço: Rua Curitiba, 832, sala 1105, Centro, Belo Horizonte - MG, 30170-120. 